\title{
Vortical susceptibility of finite-density QCD matter
}

\author{
A. Aristova, ${ }^{a}$ D. Frenklakh, ${ }^{a, c}$ A. Gorsky ${ }^{b, c}$ and D. Kharzeev ${ }^{d, e}$ \\ ${ }^{a}$ Institute of Theoretical and Experimentsl Physics, \\ B. Cheryomushkinskaya 25, Moscow, Russia \\ ${ }^{b}$ Institute of Information Transmission Problems of the Russian Academy of Sciences, \\ B. Karetny 19, Moscow, Russia \\ ${ }^{c}$ Moscow Institute of Physics and Technology, \\ Institutskii pr, Dolgoprudny, Russia \\ ${ }^{d}$ Department of Physics and Astronomy, Stony Brook University, \\ NY 11794-3800, U.S.A. \\ ${ }^{e}$ Physics Department and RIKEN-BNL Research Center, Brookhaven National Laboratory, \\ NY 11973-5000, U.S.A. \\ E-mail: aristova@itep.ru, frenklakh@itep.ru, gorsky@itep.ru, \\ dmitry.kharzeev@stonybrook.edu
}

ABSTRACT: The susceptibility of finite-density QCD matter to vorticity is introduced, as an analog of magnetic susceptibility. It describes the spin polarization of quarks and antiquarks in finite-density QCD matter induced by rotation. We estimate this quantity in the chirally broken phase using the mixed gauge-gravity anomaly at finite baryon density. It is proposed that the vortical susceptibility of QCD matter is responsible for the polarization of $\Lambda$ and $\bar{\Lambda}$ hyperons observed recently in heavy ion collisions at RHIC by the STAR collaboration.

Keywords: Spontaneous Symmetry Breaking, Chiral Lagrangians, Phase Diagram of QCD

ARXIV EPRINT: 1606.05882 


\section{Contents}

1 Introduction 1

2 Susceptibilities of the chiral condensate in the external fields 2

2.1 Magnetic susceptibility 2

2.2 Vortical susceptibility of the quark condensate 3

3 Anomalous term in the low-energy action and angular momentum 5

4 Vorticity-induced polarization throughout the hadronization: $\Lambda$ production in heavy ion collisions 5

5 Conclusion

\section{Introduction}

Finite-density relativistic matter exhibits a rich variety of macroscopic phenomena that stem from quantum anomalies involving the baryon and electric charge densities [1-3]. These anomalies lead in particular to the Chiral Vortical Effect (CVE) identified both in field theory [3] and in the holographic approach [4] (see also [5] for an early discussion); the temperature dependence of the effect is non-trivial and originates from the mixed gaugegravity anomaly $[6,7]$. The anomaly-induced effects of rotation are analogous to effects in an external magnetic field, albeit with some important differences [8].

In this paper we will extend the previous analysis to the effect of rotation on finitedensity QCD matter in the phase with spontaneously broken chiral symmetry and the corresponding gap in the excitation spectrum. The response of the ground state of a gapped system to external fields is an important property of the system. A familiar example is magnetic susceptibility of quark condensate in QCD introduced in [9] that measures the linear response of vacuum to the external magnetic field. Its anomalous nature has been recognized in [10] and it has been evaluated in holographic QCD taking into account the conventional Chern-Simons (CS) term [11] or the mixed CS term in the extended holographic model $[12,13]$.

The magnetic susceptibility of QCD in the confined phase has been evaluated on the lattice $[14,15]$ at finite temperature. It has also been shown that magnetic susceptibility is responsible for a number of interesting physical phenomena, including the T-odd correlations in dijet production [16] and the radiative decays of heavy mesons [17].

A number of physical systems possess vorticity - for example, the quark-gluon plasma produced in heavy ion collisions at a non-zero impact parameter, or dense matter in rotating neutron stars. A natural question arises: is there an analog of the magnetic susceptibility of QCD matter describing the response of the system to rotation? 
In this paper we demonstrate that there is indeed a new anomalous effect on QCD matter in spontaneously broken phase that is induced by rotation; it can be describes as a nontrivial response of the quark condensate to the external gravitational field. The simplest non-flat gravity background involves the abelian graviphoton gauge connection. The gravimagnetic curvature corresponds to the angular velocity of rotation while the gravielectric field corresponds to the temperature gradient. In this paper we will estimate the vortical susceptibility of the low density QCD matter using the anomalous triangle $\left\langle V A V_{g}\right\rangle$ where $V_{g}$ is the fermion current interacting with an external graviphoton field.

The non-vanishing vortical susceptibility of matter generates an interesting anomalous term in the low-energy QCD effective action. This new anomalous term yields the anomalous contribution to the angular momentum in the confined phase of QCD in the external rank-two field which may be called "superrotation". It is the vortical counterpart of the anomalous term in an external electromagnetic field which generates the supercurrent in QCD [18]. Recall that supercurrents in the gapped phases are specific features of the superconducting and superfluid states of matter. They are proportional to the gradient of the phase of the condensate and to the magnitude of the condensate. The chiral condensate breaks the global symmetry of the QCD Lagrangian, hence the situation is more close to the superfluid case when the global U(1) symmetry is broken. The possible superrotation in an external selfdual rank two field is of the same nature.

The paper is organized as follows. In section 2 we recall the properties of magnetic susceptibility and introduce its vortical counterpart. It is evaluated via the mixed anomaly and the pion dominance in the axial channel. In section 3 we discuss the possibilities for related effects in more general gravitational backgrounds and in the chirally imbalanced matter. The application of our results to $\Lambda$ polarization observed recently in heavy ion collisions is described in section 4 .

\section{Susceptibilities of the chiral condensate in the external fields}

\subsection{Magnetic susceptibility}

Consider the hadronic phase of QCD where the chiral symmetry is broken by the condensate $\langle\bar{\Psi} \Psi\rangle$. The linear response of the chiral condensate to an external magnetic field results in the relation

$$
\left\langle 0\left|\bar{\Psi}_{f} \sigma_{\mu \nu} \Psi_{f}\right| 0\right\rangle=e_{f} \chi_{f}\langle\bar{\Psi} \Psi\rangle F_{\mu \nu}
$$

where $\chi_{f}$ and $e_{f}$ are the susceptibility and the charge of the corresponding quark flavor $f$. Its value can be derived from the anomalous $\langle V V A\rangle$ triangle, $\chi=-N_{c} /\left(4 \pi^{2} f_{\pi}^{2}\right)$ [10].

If we introduce a physical selfdual rank-2 field $B_{\mu \nu}$, the non-vanishing magnetic susceptibility induces the following anomalous term in the chiral Lagrangian [13]

$$
L_{\mathrm{anom}}=\chi\langle\bar{\Psi} \Psi\rangle F_{\mu \nu} B_{\mu \nu} \operatorname{Tr}\left[\mathrm{BQ}\left(U+U^{-1}\right)\right],
$$

where $B_{\mu \nu}$ is the source for the fermion tensor current $\bar{\Psi} \sigma_{\mu \nu} \Psi$, B is the flavor matrix and $\mathrm{Q}$ is charge matrix. This term can be thought of as the modification of the quark mass in 
the external field. In four dimensions

$$
\sigma_{\mu \nu}=\frac{1}{2} i \epsilon_{\mu \nu \alpha \beta} \sigma_{\alpha \beta} \gamma_{5}
$$

therefore the external field has to be selfdual, $B_{\mu \nu}=\tilde{B}_{\mu \nu}$.

It has been argued in [18] that this anomalous term induces a kind of a supercurrent proportional to the condensate

$$
J_{\text {anom }, \nu}=\chi\langle\bar{\Psi} \Psi\rangle \partial_{\mu} B_{\mu \nu}
$$

which due to selfduality can be also written in terms of the dual field $\tilde{B}$. We can trade the $B_{\mu \nu}$ field for a scalar field $\Phi$; the anomalous current then acquires the form identical to the superfluid current

$$
J_{\text {superfluid }, \mu}=n_{s}\left(\partial_{\mu} \Phi-A_{\mu}\right)
$$

where $n_{s}$ is the density of the superfluid component proportional to the condensate and $\Phi$ is the condensate phase. In our case the field $\Phi$ is the scalar meson while $A_{\mu}$ is the composite current.

\subsection{Vortical susceptibility of the quark condensate}

There is a well-known analogy between an external magnetic field and the rotating frame manifested by the substitution $e_{f} \vec{B} \leftrightarrow \mu_{f} \vec{\Omega}$ that is based upon Larmor's theorem. In the relativistic case this analogy is not obvious. Nevertheless we will demonstrate that at small chemical potential (within the range of validity of the linear approximation) this substitution holds.

It is thus natural to introduce a response of the quark condensate in QCD to rotation, parametrized as

$$
\left\langle 0\left|\bar{\Psi}_{f} \sigma_{\mu \nu} \Psi_{f}\right| 0\right\rangle=\chi_{g, f}\left\langle\bar{\Psi}_{f} \Psi_{f}\right\rangle G_{\mu \nu}
$$

here we describe rotation as the curvature of an external "graviphoton" field $G_{\mu \nu}$ and denote the corresponding susceptibility as $\chi_{g, f}$. Let us emphasize that we consider the linear response approximation; in general, in dense matter there could be additional tensor structures [19].

Let us recall that the graviphoton field is introduced as the specific fluctuation of the background metric:

$$
d s^{2}=\left(1+2 \phi_{g}\right) d t^{2}-\left(1-2 \phi_{g}\right) d^{2} \vec{x}+2 \overrightarrow{A_{g}} d \vec{x} d t .
$$

The gravimagnetic field corresponds to the angular velocity of rotation at small velocity

$$
\vec{B}_{g} \propto \vec{\Omega}
$$

however at large velocities the relation between the gravimagnetic field and the angular velocity is more complicated.

To evaluate the vortical susceptibility let us first recall the derivation of magnetic susceptibility of the quark condensate in [10]. It is convenient to consider the 
$\langle V(0) V(Q) A(-Q)\rangle$ three-point function assuming that one vector vertex corresponds to the external magnetic field. This three-point function can be treated in two ways. First, assuming that $Q^{2}$ is large we can consider the OPE for the product of vector and axial currents; the first subleading term is $\left\langle m \bar{\Psi} \sigma_{\mu \nu} \Psi / Q^{4}\right\rangle$, where $\mathrm{m}$ is the quark mass. On the other hand we can assume the pion dominance in the axial channel and make use of the anomalous $\pi_{0} F \tilde{F}$ vertex. Expanding the propagator in the pion mass we get the subleading $m_{\pi}^{2} / Q^{4}$ term that has to be compared to the $Q^{-4}$ term in the OPE. The comparison of two terms and the use of the GMOR relation and (2.1) immediately yields the expression for the magnetic susceptibility $\chi=-N_{c} /\left(4 \pi^{2} f_{\pi}^{2}\right)$ found in [10].

Let us follow the same strategy in the gravimagnetic case. First let us recall that the graviphoton that is a component of the metric interacts with the energy-momentum tensor. The gravimagnetic component of the connection $A_{g}$ is coupled to the momentum of the fermion field via the term

$$
A_{g, i} \bar{\Psi}\left(\gamma_{i} D_{0}+\gamma_{0} D_{i}\right) \Psi
$$

At finite density $\mu$, there is a component of the momentum that does not involve the derivatives:

$$
\mu A_{g, i} \bar{\Psi} \gamma_{i} \Psi
$$

this expression is known from the derivation of the CVE $[6,7]$. Therefore the weak gravimagnetic field interacts with the vector current similarly to the electromagnetic current, with the additional factor $\mu$ in the vertex.

Let us now compare two ways of evaluation of the correlator: via OPE for $A(Q) T_{0 j}(0) V(-Q)$ and via the pion pole dominance in the axial channel, similarly to [10]. Since the momentum in $T_{0 j}$ vertex vanishes, the operator in the case if finite density QCD reduces to the vector current multiplied the chemical potential $\mu$; therefore we get the correlator that is similar to the magnetic case. From the OPE side we get the same operator $m \bar{\Psi} \sigma_{\mu \nu} \Psi / Q^{4}$ as the first subleading term - therefore the OPE involves precisely the matrix element we are looking for.

From the pion pole side we will now use the anomalous pion-gravity $\pi_{0} V B_{g}$ vertex and expand the pion propagator in pion mass once again. The specific vertex which involves the Goldstone particle, electromagnetic field and the graviphoton field exists only in dense matter $[1,2]$ and reads as

$$
\delta L=-N_{C} \sum_{f} \frac{e_{f} \mu_{f}}{4 \pi^{2}} \epsilon^{\mu \nu \alpha \beta} \partial_{\mu} \pi A_{\alpha} \partial_{\nu} A_{g, \beta}
$$

This vertex allows us to make the comparison with the OPE calculation. The leading term corresponds to the pion pole, and comparison of the two $Q^{-4}$ terms yields

$$
\chi_{g, f}=\frac{N_{c} \mu_{f}}{\pi^{2} f_{\pi}^{2}}
$$

where we have introduced the susceptibility for each flavor. Of course, as in the magnetic case, the assumption of pion dominance brings in an uncertainty due to the omission of excited resonances; however we believe that this result provides a reasonable estimate. 


\section{Anomalous term in the low-energy action and angular momentum}

What are the possible observable manifestations of the vortical susceptibility of the condensate? To answer this question let us first note that the non-vanishing vortical susceptibility of the chiral condensate implies the following anomalous term in the low-energy chiral Lagrangian:

$$
L_{\text {anom }}=\chi_{g}\langle\bar{\Psi} \Psi\rangle G_{\mu \nu} B_{\mu \nu} \operatorname{Tr} B\left(U+U^{-1}\right),
$$

where $\mathrm{B}$ is the flavor matrix (the charge matrix does not enter this expression). In the gravimagnetic case when $G_{i j}=2 \epsilon_{i j k} \Omega_{k}$ instead of the anomalous current we get the anomalous angular momentum induced by the external rank-two B field

$$
J_{i} \propto \chi_{g}\langle\bar{\Psi} \Psi\rangle \epsilon_{i j k} B_{j k} .
$$

As noted before, the rank-two B field is selfdual and can be traded for the scalar and the composite current.

So far we have found the vortical susceptibility of the quark condensate which is proportional to the vector chemical potential. One may also consider the case of the chirally imbalanced matter with chiral chemical potential $\mu_{5} \neq 0$. In this case due to the parity-odd nature of the chemical potential several new effects emerge. First, one can introduce the susceptibility in the external gravielectric field that describes the temperature gradient:

$$
\left\langle 0\left|\bar{\Psi} \sigma_{\mu \nu} \Psi\right| 0\right\rangle=\tilde{\chi}_{g}\langle\bar{\Psi} \Psi\rangle \tilde{G}_{\mu \nu},
$$

where the $\tilde{\chi}_{g} \propto \mu_{5}$ and the gravielectric field $E_{g} \propto \nabla \log T$.

In addition, in chirally imbalanced matter we expect the "anomalous boost" induced by the external rank-two field and given by the derivative of the Lagrangian with respect to the gravieletric field $G_{0 j}$ :

$$
J_{j}=\frac{\delta L}{\delta G_{0 j}} .
$$

This vector is dual to the angular momentum which is the axial vector. It is proportional to the dual vortical susceptibility and the corresponding component of the rank 2 external field:

$$
J_{j} \propto \tilde{\chi}_{g}\langle\bar{\Psi} \Psi\rangle B_{0 j}
$$

The chiral chemical potential can be generated by topological QCD fluctuations, or by a time-dependent axion field during the creation and decay of the extended axionic defects like the axion string and axion domain wall.

\section{Vorticity-induced polarization throughout the hadronization: $\Lambda$ pro- duction in heavy ion collisions}

The vortical susceptibility of the quark condensate discussed above has a simple physical interpretation: vorticity aligns the spins of quarks and antiquarks along the axis of rotation. While the alignment of quark spins by rotation in a chirally symmetric phase has been extensively discussed in the literature [3, 21-28] (see [29] for a recent review), a priori 
it is not clear whether any of this alignment would survive the hadronization. Indeed, hadronization is accompanied by the spontaneous breaking of chiral symmetry, and could thus easily wash out any spin polarization present in the chirally symmetric phase. Our results in this paper however indicate that the polarization of quarks and antiquarks induced by vorticity should survive the hadronization transition. This effect should manifest itself in spin polarization of the observed hadrons.

Recently, the STAR collaboration at RHIC has presented the preliminary data indicating that both $\Lambda$ and $\bar{\Lambda}$ hyperons in AuAu collisions at $\sqrt{s}=62 \mathrm{GeV}$ and below appear polarized relative to the reaction plane, with $P_{\Lambda} \simeq P_{\bar{\Lambda}} \simeq 0.02-0.03[30,31]$. We believe that this observation is remarkable for the following reasons: i) it presents a clear evidence for the presence of vorticity in the quark-gluon fluid; ii) it shows that vorticity is still present at the hadronization transition, suggesting a small value of shear viscosity; iii) it demonstrates that the strange quark and antiquark polarization survives the hadronization.

Let us now present the estimate of $\Lambda$ and $\bar{\Lambda}$ polarization basing on our results. Assume that the vorticity is aligned along the $z$ axis; the average spin polarization of quarks and antiquarks from (2.6) and (2.12) is

$$
\left\langle\sigma_{z}\right\rangle=\frac{\left\langle\bar{\Psi} \sigma_{x y} \gamma_{5} \Psi\right\rangle}{\langle\bar{\Psi} \Psi\rangle}=\frac{N_{c}}{\pi^{2} f_{\pi}^{2}} \mu \Omega_{z}
$$

where $\mu$ is the baryon chemical potential and $\Omega$ is the vorticity.

For a numerical estimate, let us use the baryon chemical potential $\mu \simeq 80 \mathrm{MeV}$ as extracted from hadron abundances in AuAu collisions at $\sqrt{s}=62 \mathrm{GeV}$ [32]. Since the polarization of $\Lambda$ hyperons results mostly from the polarization of strange quarks, we have to use the chemical potential of strange quarks that is $\mu_{s} \simeq \mu / 3$. The value of vorticity on the freeze-out hyper-surface at present is somewhat uncertain. The lower bound on this quantity can be obtained by making a (somewhat unrealistic) assumption that no vorticity is present in the initial conditions. One can then use viscous $(3+1)$ relativistic hydrodynamics with shear viscosity to entropy ratio $\eta / s=0.1$ to obtain the lower bound of $\Omega_{z} \simeq 5 \cdot 10^{-3} \mathrm{fm}^{-1}$ [33]. On the other hand, the evaluation of vorticity in the initial state [34] from HIJING model yields for initial vorticity a large value $\Omega_{z}^{0} \simeq 0.1$. Hydrodynamical evolution at small shear viscosity is then expected to approximately preserve vorticity, yielding the value of $\Omega_{z} \simeq 0.1 \mathrm{fm}^{-1}$ on the freeze-out hypersurface [35].

With these values $\left(\mu_{s} \simeq 27 \mathrm{MeV}, \Omega_{z} \simeq 0.1 \mathrm{fm}^{-1}\right), N_{c}=3$ and the pion decay constant $f_{\pi}=92 \mathrm{MeV}$ we get from (4.1) the strange quark polarization

$$
P_{s} \equiv\left\langle\sigma_{z}\right\rangle \simeq 0.02
$$

Of course, the polarization of $\Lambda$ and $\bar{\Lambda}$ will be suppressed by the dilution factor $D$ relative to the polarization of strange quarks and antiquarks:

$$
P_{\Lambda}=P_{\bar{\Lambda}}=D P_{s} .
$$

The data on production of polarized $\Lambda$ and $\bar{\Lambda}$ hyperons in semi-inclusive Deep-Inelastic scattering allow to extract the dilution factor; in the kinematic region of valence quarks appropriate for our case, it is $D=0.9 \pm 0.2$ [36]. 
We thus estimate

$$
P_{\Lambda}=P_{\bar{\Lambda}} \simeq 0.02,
$$

in reasonable agreement with STAR data [30, 31]. Of course, this agreement could be much worse if we assumed the absence of vorticity in the initial state. We think however that this assumption is unrealistic; clearly, much more work has to be done to control the magnitude of vorticity.

\section{Conclusion}

In this paper we have introduced the vortical susceptibility of finite density matter in the chirally broken phase of QCD. It originates from the anomaly, and has been estimated here under the assumption of pion dominance in the axial channel. The non-vanishing vortical susceptibility signals a kind of "super-rotation" (anomalous contribution to the angular momentum) present in a background antisymmetric tensor or axion fields in the rotating frame. It would be interesting to investigate the vortical susceptibility on the lattice similarly to how it has been done previously for magnetic susceptibility. This quantity can also be evaluated in the holographic approach.

We discuss a macroscopic property of the QCD matter, and it would be interesting to establish the microscopic origin of gravimagnetization in the rotating frame. Since the vortical susceptibility is proportional to the baryon density it is natural to assume that at microscopic level one deals with gravimagnetization of the degrees of freedom that carry baryon charge. This can be compared with the gravimagnetization of the vacuum in the $N=2 \mathrm{SYM}$ theory when microscopically the gravimagnetization of vacuum is dominated by the instantons [20] and can be evaluated from Nekrasov partition function at small parameters of the Omega-deformation. Recall that baryons are identified as the instantons in the holographic QCD hence the SYM results could provide some guidance in understanding gravimagnetization in QCD vacuum.

It would also be interesting to consider the vortical susceptibility beyond the linear response approximation. In analogy with the magnetic field case one may expect a saturation of vortical susceptibility at some value of vorticity; at very large vorticity, the QCD matter has recently been argued [28] to undergo transition to the chirally symmetric phase. Another interesting direction is the application of these ideas in condensed matter physics, to systems possessing exciton condensates. The exciton condensate is the analog of the chiral condensate, so one can address the response of the exciton condensate to the rotation of the system.

\section{Acknowledgments}

We thank A. Abanov and N. Sopenko for discussions on vortical susceptibility, and F. Becattini, X. Guo, X.-G. Huang, J. Liao, and M. Lisa for valuable exchanges on the role of vorticity in heavy ion collisions. A.G. thanks the Simons Center for Geometry and Physics at Stony Brook University where this paper has been completed for hospitality and support during the program "Geometry of the Quantum Hall State". D.K. acknowledges support 
of the Alexander von Humboldt foundation and Le Studium foundation, Loire Valley, France for support during the "Condensed matter physics meets relativistic quantum field theory" program. The work of D.K. was supported by the U.S. Department of Energy under Contracts No. DEFG-88ER40388 and DE-SC0012704. The work of A.A., D.F. was supported by grant RFBR - 15-02-02092, the work of A.G. by grant of Russian Science Foundation 14-050-00150 for IITP.

Open Access. This article is distributed under the terms of the Creative Commons Attribution License (CC-BY 4.0), which permits any use, distribution and reproduction in any medium, provided the original author(s) and source are credited.

\section{References}

[1] D.T. Son and A.R. Zhitnitsky, Quantum anomalies in dense matter, Phys. Rev. D 70 (2004) 074018 [hep-ph/0405216] [InSPIRE].

[2] M.A. Metlitski and A.R. Zhitnitsky, Anomalous axion interactions and topological currents in dense matter, Phys. Rev. D 72 (2005) 045011 [hep-ph/0505072] [INSPIRE].

[3] D. Kharzeev and A. Zhitnitsky, Charge separation induced by P-odd bubbles in QCD matter, Nucl. Phys. A 797 (2007) 67 [arXiv:0706.1026] [INSPIRE].

[4] J. Erdmenger, M. Haack, M. Kaminski and A. Yarom, Fluid dynamics of R-charged black holes, JHEP 01 (2009) 055 [arXiv:0809.2488] [INSPIRE].

[5] A. Vilenkin, Macroscopic parity violating effects: neutrino fluxes from rotating black holes and in rotating thermal radiation, Phys. Rev. D 20 (1979) 1807 [INSPIRE].

[6] K. Landsteiner, E. Megias and F. Pena-Benitez, Gravitational anomaly and transport, Phys. Rev. Lett. 107 (2011) 021601 [arXiv:1103.5006] [INSPIRE].

[7] K. Landsteiner, E. Megias, L. Melgar and F. Pena-Benitez, Holographic gravitational anomaly and chiral vortical effect, JHEP 09 (2011) 121 [arXiv:1107.0368] [INSPIRE].

[8] D.E. Kharzeev and D.T. Son, Testing the chiral magnetic and chiral vortical effects in heavy ion collisions, Phys. Rev. Lett. 106 (2011) 062301 [arXiv: 1010.0038] [INSPIRE].

[9] B.L. Ioffe and A.V. Smilga, Nucleon magnetic moments and magnetic properties of vacuum in QCD, Nucl. Phys. B 232 (1984) 109 [INSPIRE].

[10] A. Vainshtein, Perturbative and nonperturbative renormalization of anomalous quark triangles, Phys. Lett. B 569 (2003) 187 [hep-ph/0212231] [INSPIRE].

[11] A. Gorsky and A. Krikun, Magnetic susceptibility of the quark condensate via holography, Phys. Rev. D 79 (2009) 086015 [arXiv: 0902.1832] [inSPIRE].

[12] S.K. Domokos, J.A. Harvey and A.B. Royston, Successes and failures of a more comprehensive hard wall AdS/QCD, JHEP 04 (2013) 104 [arXiv: 1210.6351] [INSPIRE].

[13] A. Gorsky, P.N. Kopnin, A. Krikun and A. Vainshtein, More on the tensor response of the QCD vacuum to an external magnetic field, Phys. Rev. D 85 (2012) 086006 [arXiv: 1201.2039] [INSPIRE].

[14] P.V. Buividovich, M.N. Chernodub, E.V. Luschevskaya and M.I. Polikarpov, Chiral magnetization of non-Abelian vacuum: a lattice study, Nucl. Phys. B 826 (2010) 313 [arXiv: 0906.0488] [INSPIRE]. 
[15] G.S. Bali et al., Magnetic susceptibility of QCD at zero and at finite temperature from the lattice, Phys. Rev. D 86 (2012) 094512 [arXiv: 1209.6015] [inSPIRE].

[16] V.M. Braun, S. Gottwald, D. Yu. Ivanov, A. Schafer and L. Szymanowski, Exclusive photoproduction of hard dijets and magnetic susceptibility of QCD vacuum, Phys. Rev. Lett. 89 (2002) 172001 [hep-ph/0206305] [INSPIRE].

[17] J. Rohrwild, Determination of the magnetic susceptibility of the quark condensate using radiative heavy meson decays, JHEP 09 (2007) 073 [arXiv:0708.1405] [INSPIRE].

[18] A. Gorsky and D. Kharzeev, On the supercurrent in QCD vacuum, to appear.

[19] H. Itoyama and A.H. Mueller, The axial anomaly at finite temperature, Nucl. Phys. B 218 (1983) 349 [inSPIRE].

[20] A. Gorsky, Angular momentum and gravimagnetization of the $N=2 S Y M$ vacuum, Theor. Math. Phys. 171 (2012) 616 [arXiv:1102.1841] [INSPIRE].

[21] D. Kharzeev, Parity violation in hot QCD: why it can happen and how to look for it, Phys. Lett. B 633 (2006) 260 [hep-ph/0406125] [INSPIRE].

[22] Z.-T. Liang and X.-N. Wang, Globally polarized quark-gluon plasma in non-central $A+A$ collisions, Phys. Rev. Lett. 94 (2005) 102301 [Erratum ibid. 96 (2006) 039901] [nucl-th/0410079] [INSPIRE].

[23] Z.-T. Liang and X.-N. Wang, Spin alignment of vector mesons in non-central $A+A$ collisions, Phys. Lett. B 629 (2005) 20 [nucl-th/0411101] [INSPIRE].

[24] B. Betz, M. Gyulassy and G. Torrieri, Polarization probes of vorticity in heavy ion collisions, Phys. Rev. C 76 (2007) 044901 [arXiv:0708.0035] [InSPIRE].

[25] J.-H. Gao, S.-W. Chen, W.-T. Deng, Z.-T. Liang, Q. Wang and X.-N. Wang, Global quark polarization in non-central A+A collisions, Phys. Rev. C 77 (2008) 044902 [arXiv:0710.2943] [INSPIRE].

[26] O. Rogachevsky, A. Sorin and O. Teryaev, Chiral vortaic effect and neutron asymmetries in heavy-ion collisions, Phys. Rev. C 82 (2010) 054910 [arXiv: 1006.1331] [INSPIRE].

[27] F. Becattini, L. Csernai and D.J. Wang, $\Lambda$ polarization in peripheral heavy ion collisions, Phys. Rev. C 88 (2013) 034905 [Erratum ibid. C 93 (2016) 069901] [arXiv:1304.4427] [INSPIRE].

[28] Y. Jiang and J. Liao, Pairing phase transitions of matter under rotation, arXiv:1606.03808 [INSPIRE].

[29] D.E. Kharzeev, J. Liao, S.A. Voloshin and G. Wang, Chiral magnetic and vortical effects in high-energy nuclear collisions - a status report, Prog. Part. Nucl. Phys. 88 (2016) 1 [arXiv: 1511.04050] [INSPIRE].

[30] STAR collaboration, M. Lisa et al., Global polarization of Lambda hyperons from STAR, talk at QCD Workshop on Chirality, Vorticity and Magnetic field in Heavy Ion Collisions, UCLA, U.S.A. January 2016.

[31] STAR collaboration, H. Huang et al., Experimental overview on the search of anomalous chiral effects, talk at the BEST 2016 Workshop, Indiana University, U.S.A. May 2016.

[32] P. Braun-Munzinger, V. Koch, T. Schäfer and J. Stachel, Properties of hot and dense matter from relativistic heavy ion collisions, Phys. Rept. 621 (2016) 76 [arXiv:1510.00442] [INSPIRE]. 
[33] F. Becattini et al., A study of vorticity formation in high energy nuclear collisions, Eur. Phys. J. C 75 (2015) 406 [arXiv:1501.04468] [InSPIRE].

[34] W.-T. Deng and X.-G. Huang, Vorticity in heavy-ion collisions, Phys. Rev. C 93 (2016) 064907 [arXiv: 1603.06117] [INSPIRE].

[35] X. Guo, X.G. Huang and D. Kharzeev, work in progress.

[36] J.R. Ellis, D. Kharzeev and A. Kotzinian, The proton spin puzzle and $\Lambda$ polarization in deep inelastic scattering, Z. Phys. C 69 (1996) 467 [hep-ph/9506280] [INSPIRE]. 